\title{
SleepSense: Non-invasive Sleep Event Recognition Using An Electromagnetic Probe
}

\author{
Yan Zhuang ${ }^{1}$, Chen Song ${ }^{1}$, Aosen Wang ${ }^{1}$, Feng Lin $^{1}$, Yiran $\mathrm{Li}^{2}$, Changzhan $\mathrm{Gu}^{2}$, Changzhi $\mathrm{Li}^{2}$, Wenyao $\mathrm{Xu}^{1}$ \\ 1 Department of Computer Science and Engineering, SUNY at Buffalo, New York, USA \\ 2 Department of Electrical and Computer Engineering, Texas Tech University, Texas, USA \\ Email: \{yanzhuan, csong5, aosenwan, flin28, wenyaoxu\}@buffalo.edu \\ \{yiran.li, changzhan.gu, changzhi.li\}@ttu.edu
}

\begin{abstract}
Sleep monitoring is receiving increased attention in the healthcare community, because the quality of sleep has a great impact on human health. Existing in-home sleep monitoring devices are either obtrusive to the user or cannot provide adequate sleep information. To this end, we present SleepSense, a contactless and low-cost sleep monitoring system for home use that can continuously detect the sleep event. Specifically, SleepSense consists of three parts: an electromagnetic probe, a robust automated radar demodulation module, and a signal processing framework for sleep event recognition, including on-bed movement, bed exit, and breathing event. We present a prototype of the SleepSense system, and perform a set of comprehensive experiments to evaluate the performance of sleep monitoring. Using a real-case evaluation, experimental results indicate that SleepSense can perform effective sleep event detection and recognition in practice.
\end{abstract}

\section{INTRODUCTION}

The quality of sleep has a great impact on human health. There is a growing recognition of adverse effects from poor sleep quality and sleep disorders. Subjects with sleep disorders are more likely to suffer from chronic diseases, such as heart disease, diabetes, and hypertension. Moreover, people are usually not being aware of sleep disorders because they happen during sleep. Currently, it has became a chronic, underexplored but critical health challenge in the modern life.

To date, polysomnography (PSG) is still the primary and the most objective sleep assessment method in clinical use [12]. An alternative sleep quality estimation method is actigraphy, including an accelerometer and a memory storage chip, which can provide information on movements during sleep. Recent research is seeking for new sleep monitoring methods and has proposed several sleep monitoring approaches. Rofouei et al. [11] presented a wearable neck-cuff system for sleep monitoring. Wang et al. [13] developed a video system to monitor sleep. Hao et al. [3] presented a smart phone microphone-based sleep quality monitoring system. Liu et al. [8] designed a bedsheet that comprises arrays of pressure sensors to monitor the sleep posture. This system also can unobtrusively monitor the breathing pattern during the sleep [7]. Nevertheless, current sleep monitoring methods suffer from several drawbacks, such as invasiveness, lack of privacy, and high implementation cost. For instance, subject wears the monitoring devices, which cause the uncomfortable feeling and decrease sleep quality during sleep; recording the raw video and audio signal from user raises their concerns about privacy; high-cost limits the wide accessibility. These limitations prevent people from the existing sleep monitoring systems.

To address the above challenges, we present SleepSense, a contactless and low-cost sleep monitoring system. We design and implement an electromagnetic probe based on the commercial off-the-shelf (COTS) components, which is able to accurately capture the sleep-related signal. Then, the sampled baseband signal goes through a demodulation module that uses the extended differentiate and cross multiple (DACM) algorithm to obtain sleep-related displacement signal. Moreover, the sleep event recognition framework processes the displacement signal and recognizes three sleep events, i.e, onbed movements, bed exits, and breathing events. Meanwhile, the breathing rate is extracted via a novel breathing rate extracting algorithm. Finally, the system is evaluated by a set of real-case studies. Specifically, in short-term controlled study, SleepSense demonstrates the recognition rate for breathing event, on-bed movement, and bed exit detection, which is $98.8 \%, 88.6 \%$, and $67.7 \%$, respectively. The 75-minute nap study demonstrates the SleepSense's high accuracy rate for sleep event recognition and wide usability in the real world.

The rest of paper is organized as follows: Section II presents the overview of SleepSense. Section III describes the implementation of SleepSense, including the electromagnetic probe, the baseband signal demodulation module, and a sleep event recognition framework. In Section IV, we perform sets of experiments to evaluate the SleepSense. Finally, Section V concludes the paper and describes the future work.

\section{SySTEM OVERVIEW}

SleepSense is a contactless and cost-effective sleep monitoring system, which continuously detects the sleep event and obtains the breathing rate. Fig. 1 depicts the overall system architecture of SleepSense. First, the electromagnetic probe captures the sleep-related signal from the subject and outputs the baseband signal $I / Q$. Then, the demodulation layer employs an extended DACM demodulation algorithm to obtain the displacement signal $x(t)$. The sleep event recognition framework, finally, extracts the sleep-event related features from the displacement signal $x(t)$ and classifies these sleep events. In the meanwhile, a novel breathing rate extraction algorithm calculates the respiration rate. 
(1)

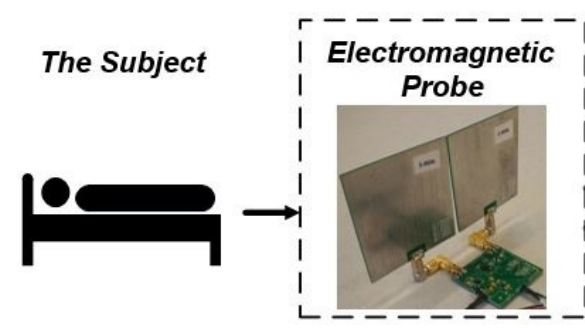

(2)

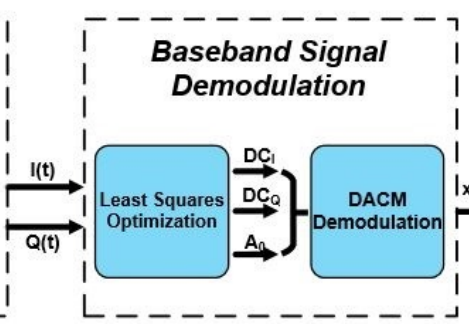

(3)

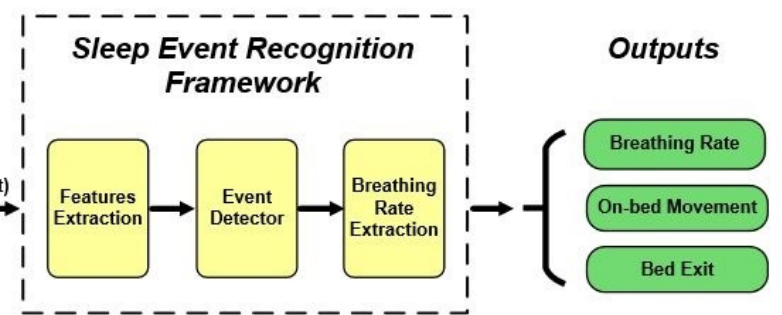

Fig. 1. The block diagram of SleepSense: (1) electromagnetic probe; (2) baseband signal demodulation module; (3) sleep event recognition framework.

\section{System Design}

\section{A. Sleep Events of Interest}

Sleep is a period of inactivity and rejuvenation. During sleep, the breathing pattern and movement distribution pattern are particularly being interested, as they characterize the different sleep states [1] [15] and are closely associated the sleep quality. Based on the movement distribution pattern and breathing pattern, we define three sleep events: on-bed movement event, bed exit event, and breathing event. The onbed movement event contains the movements such as turn over and arm trebling. In the breathing event, the subject keeps still on the bed without body movements. The bed exit event refers to the event with bed exit movement, which indicates the interruption of sleeping state. Successful recognition of these three interested sleep events is the basis for obtaining the breathing pattern and movement distribution pattern.

\section{B. Electromagnetic Probe}

The purpose of our proposed system is to perform sleep monitoring in a remote and cost-effective manner. This aim is achieved by using an electromagnetic probe. In the past years, the electromagnetic probe is employed to detect the human vital signs, such as respiration and heartbeat. Huang et al. [4] presented the feasibility of detecting heartbeat using a electromagnetic sensor. Droitcour et al. [2] and Lubecke et al. [10] have reported some good progress on sensing the respiration signs. Likewise, other researchers also use the electromagnetic probe to detect the human motion, such as hand gesture recognition [17] and fall detection [9]. In our case, we design and build this electromagnetic probe based on the COTS components, which also can sense human motions. Specifically, the electromagnetic probe generates a single-tone carrier signal which is transmitted to the subject. When the microwave hits the subject, the body displacement (caused by the movement or the respiration) of the subject enables the microwave to generate a phase shift. This phase shift is proportional to the corresponding body displacement. By demodulating this phase information properly, we can obtain the displacement information.

Fig. 2 depicts our electromagnetic probe and its block diagram. The electromagnetic probe adopts direct-conversion radar architecture to capture the subject movement and breathing signal. In the circuit implementation, as shown in Fig. 2(b), the voltage controlled oscillator (VCO) in the transmitter generates a carrier signal at $2.4 \mathrm{GHz}$ space. The VCO also provides local oscillator (LO) to the mixer in the receiver chain. The output power of this transmitter is around $0 \mathrm{dBm}$. A low
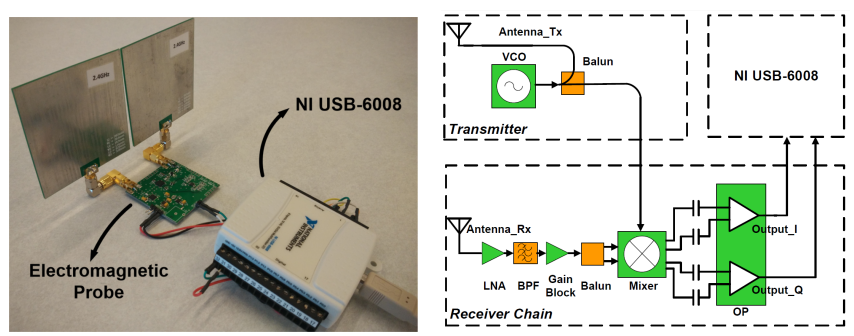

(a) The electromagnetic probe and (b) The block diagram of electromagthe DAQ NI-USB 6008 device. netic probe.

Fig. 2. The electromagnetic probe and a DAQ device. The electromagnetic probe captures the sleep-related signal and outputs the baseband signal, which is digitized by a DAQ device.

noise amplifier (LNA), a band pass filter (BPF), a gain block, a balun, a mixer, and two baseband operational amplifiers (OPs) form the receiver chain. The LNA amplifies the received signal at $2.4 \mathrm{GHz}$. The interferences with frequencies outside the 2.4 $\mathrm{GHz}$ band is removed by the BPF. A gain block is adopted to further amplify the received signal. Two OPs with the same gain of $40 \mathrm{~dB}$ are used to amplify the down-converted $I(t)$ and $Q(t)$ baseband signals [6]. Lastly, a NI data acquisition devices (DAQ), NI USB-6008, digitizes the baseband $I(t)$ and $Q(t)$ signal.

\section{Electromagnetic Probe Baseband Signal Demodulation}

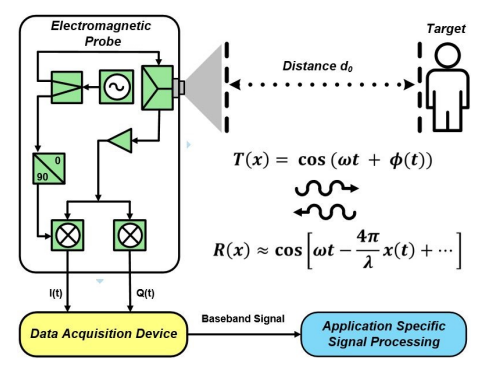

Fig. 3. The principle of electromagnetic probe application.

Fig. 3 briefly illustrates the basic principle of electromagnetic probe-based application. The electromagnetic probe transmits the continuous wave signal $T(t)$ and receives the transmission wave $R(t) . R(t)$, then, generates two baseband signals $I(t)$ and $Q(t) . I(t)$ is the in-phase signal and $Q(t)$ is the quadrature signal. In our work, for the simplicity, the $I(t)$ and $Q(t)$ can be described as in Eq. (1):

$$
\left\{\begin{array}{l}
I(n)=A_{0} \cos \left(\frac{4 \pi x(n)}{\lambda}\right)+D C_{I} \\
Q(n)=A_{0} \sin \left(\frac{4 \pi x(n)}{\lambda}\right)+D C_{Q}
\end{array} .\right.
$$


According to the trigonometric identities, the samples of $I / Q$ channels stay on a circle whose center is $\left(D C_{I}, D C_{Q}\right)$ with a radius of $A_{0}$. Given the measurement of $I(t)$ and $Q(t)$, we identify the three unknown parameters: $D C_{I}, D C_{Q}$, and $A_{0}$ using the least square optimization method [16]. Then, we employ an extended DACM algorithm proposed by Wang et $a l$. [14] to obtain the displacement signal $x(t)$. This extended DACM algorithm, as described in Eq. (2), avoids the discontinuity problem and is robust to the random noise.

$$
\Phi[n]=\sum_{k=2}^{n} \frac{I[k]\{Q[k]-Q[k-1]\}-\{I[k]-I[k-1]\} Q[k]}{I[k]^{2}+Q[k]^{2}} .
$$

\section{Sleep Event Recognition Framework}

We present the sleep event recognition framework in this section. Fig. 4 shows the architecture of this framework, including signal segmentation, features extraction, sleep event recognition, and a breathing rate extraction algorithm. First, the displacement signal $x(t)$ is framed into short segments with the window length of 512 samples (sampling rate is $100 \mathrm{~Hz}$ ). This method of segmentation is favored since it well associates the segment with the body movement in one frame and benefits further processing. Then, the framework extracts the features for each segment and classifies these segments into three sleep events: on-bed movement event, bed exit event, or breathing event. Simultaneously, breathing rate is obtained by using a novel breathing rate extraction algorithm.

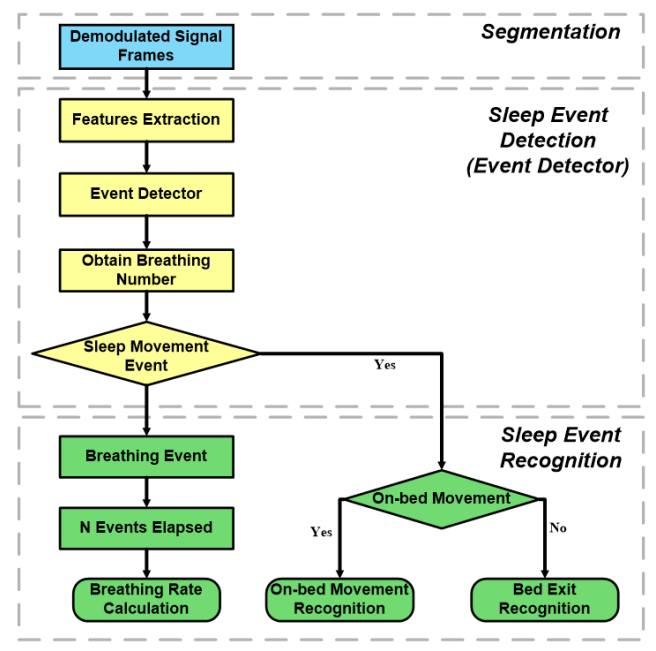

Fig. 4. The flow chart of sleep event recognition framework, consisting of segmentation, sleep event detection, and sleep event recognition.

1) Features Extraction: Our defined sleep events are characterized with a sort of time-domain and frequency-domain features. For instance, the breathing event has the subtle fluctuation in the displacement signal $x(t)$ in terms of amplitude and frequency, while the on-bed movement event and bed exit event have the relatively larger change. To represent these three different sleep events, we, therefore, extract five features including two statistical features, two frequencydomain features, and a non-linear time-series feature, i.e., root mean square (RMS), mean crossing rate (MCR), energy, mel-frequency cepstral-based coefficients (MFCC-based coefficients), and sample entropy, which are shown in Table I.

RMS is a parameter that implies the size of signal amplitude, which is the strength of the subject's movement in our case. The RMS value varies when a dramatic sleep movement happens. MCR, on the other hand, is the expected rate where the demodulated signal $x(t)$ passes the mean value, representing the changing rate in frequency. To be specific, it counts the times when the value is above or under the mean value. In our cases, the on-bed movement and bed exit movement will produce the dramatic fluctuation in terms of frequency. Therefore, the MCR value are various for these three sleep events. Energy is a common frequency-domain feature in the signal processing field. Obviously, the event with movements, such as bed exit movement and on-bed movement, has larger energy than that of the breathing event. Another commonly used frequency domain feature for analysis is MFCC, especially in the audio signal processing area. Hughes et al. [5] and Liu et al. [9] has proved that MFCC features are effective to represent the Doppler signatures and be employed to recognize various human activities. Likewise, we also compute MFCC features for each segment. However, unlike the traditional MFCC technology, which uses an melfilter bank to block the signal in a certain frequency range. In our case, we do not implement the mel-filter banks, because the frequency features of on-bed movement, bed exit, and breathing event are in all frequency domain. Therefore, we must keep all the frequency features in all frequency domain, and no filter is needed. Finally, we extract the sample entropy for each frame as the last feature, because the sample entropy provides an enhanced method to measure the complexity and regularity of the signal. In our case, the displacement signal of breathing event has more regularity and less complexity than the displacement signal of the on-bed movement and bed exit event, thus having a distinguished sample entropy value. After obtaining these feature vectors, the framework uses a decision tree-based sleep event detector to separate the these sleep events.

2) Breathing Rate Extraction: In this section, we present the breathing rate calculation algorithm by using the novel peak detection with adaptive threshold. The breathing signal is similar with the trigonometric signal. A pair of peak and valley is corresponding to a breathing event. Therefore, to extract the breathing rate in the displacement signal $x(t)$, the number of pairs of peak and valley in one segment is counted. Traditionally, this aim can be achieved by employing a general peak detection method with the fixed threshold. The threshold is defined as the difference between the peak (or valley) and its neighboring values. However, during sleep, the signal of breathing and sleep movement from different subjects usually vary in magnitude and frequency, resulting in the inaccurate peak detection if it employs the traditional threshold-fixed peak finding method. Therefore, a new peak detection algorithm with adaptive threshold is presented to resolve this issue.

When a segment of displacement signal $x(n)$ arrives, the proposed breathing rate extraction algorithm obtains the relationship between the breathing number and the threshold value. Specifically, this algorithm goes through all the threshold values from 0 to the maximal magnitude difference, and the corresponding number of peaks (or valleys) is detected, as 
TABLE I. THE LIST OF SELECTED FEATURES.

\begin{tabular}{|c|c|c|c|}
\hline No. & Feature List & Feature Definition & Main Contained Info. \\
\hline 1 & RMS & The quadratic mean value of the signal & Statistical information \\
\hline 2 & MCR & of changes between below mean \& above mean & Statistical information \\
\hline 3 & Energy & The energy over the window & Frequency-domain information \\
\hline 4 & MFCC-based Coefficients & The MFCC-based coefficients & Frequency-domain information \\
\hline 5 & Sample Entropy & The regularity and complexity of the distribution of the signal & Non-linear time-domain information \\
\hline
\end{tabular}

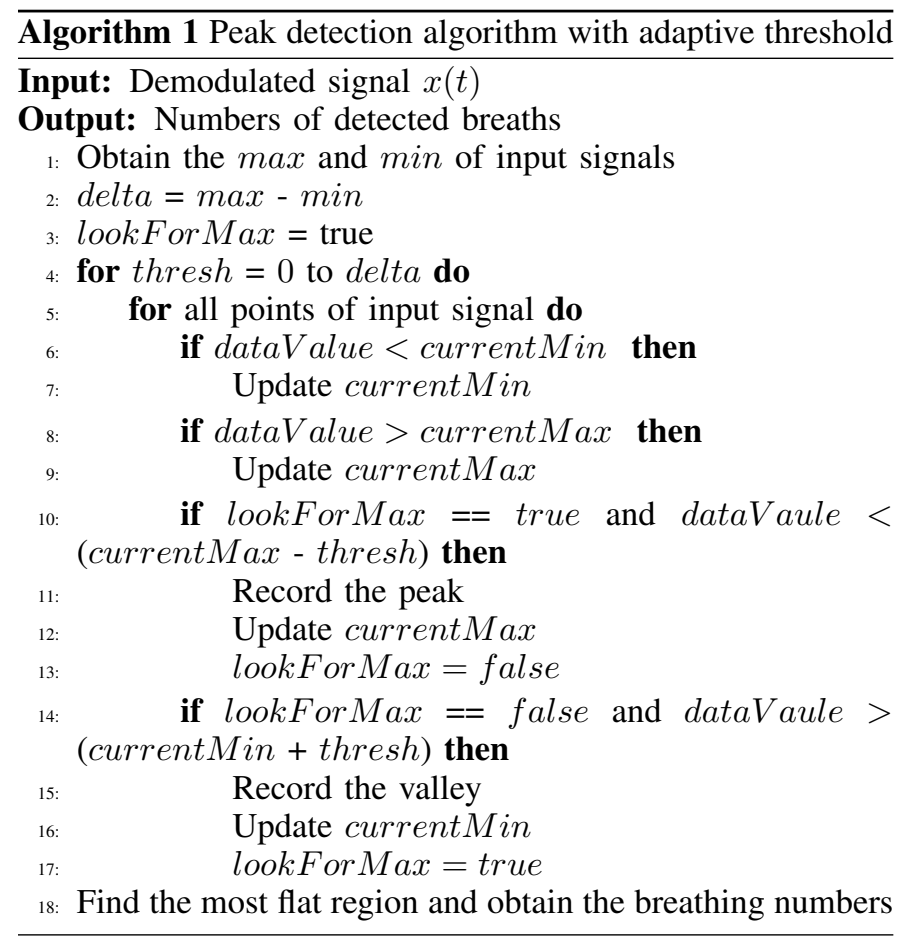

shown in Fig. 5. In Region $A$ and Region $C$, where the breathing number varies largely according to the threshold index. It means that the detected breathing number experience a large variability when the threshold value changes with a small value, representing the sensitivity to the random noise. However, in the most flat Region $B$, the breathing number stay unchanged when the thresholds increases, which implies that most of the random noise is ignored and only the true peaks are countered. Therefore, the breathing number, which is corresponding to the most flat area, is obtained. The description of breathing rate extraction algorithm is explained in detail in Algorithm1.

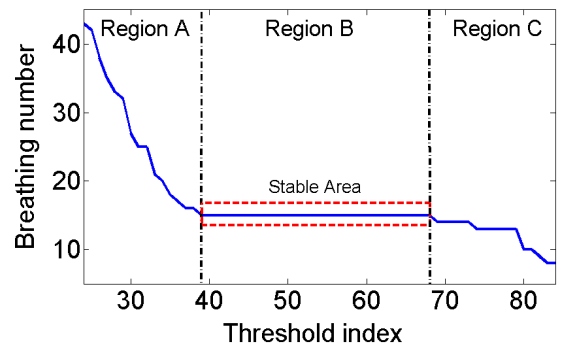

Fig. 5. A peak detection with adaptive threshold example. In this example, region $A$ and region $C$ have the inconsistent breathing numbers, implying it is sensitive to the random noise. However, region $B$ corresponding with the most flat area has the most consistent values, which are the actual peak numbers.

\section{EXPERIMENTS}

In this work, we perform several experiments to evaluate SleepSense. There are two sets of experiments. First, we have a short-term sleep study to quantify the system performance in a controlled setup. Second, we conduct a long-term sleep study, which focuses on the system usability in a naturalistic setup.

\section{A. Experimental Setup}

In the experiment, the subject lies on a mattress, as shown in Fig. 6. The electromagnetic probe is located on the top of subject with the distance of one meter. For simplicity, we employ a NI DAQ device (NI USB-6008) to sample the baseband signal at $100 \mathrm{~Hz}$. This NI DAQ device can be replaced by any other DAQ devices or self-customized DAQ devices to reduce the implementation cost. Simultaneously, an airflow sensor and an accelerometer provide the ground truth signal for the breathing and movement signal.

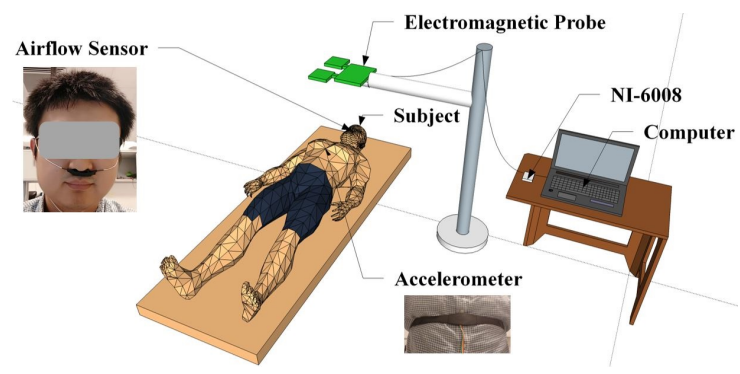

Fig. 6. The experimental setup: the subject lies his back on a mattress. The electromagnetic probe locates on the top of the chest with the distance of one meter. In the meanwhile, an airflow sensor and an accelerometer sensor are attached to serve as the ground truth measurements.

\section{B. Quantitative System Performance Evaluation}

This short-term controlled study aims to qualitatively examine the performance of SleepSense system.

1) Data Collection: The data collection in this section follows the experimental configuration in section IV-A. Three subjects participated in the short-term controlled study. Their ages are 25 to 28 years, heights 180 to $185 \mathrm{~cm}$, weight $80 \mathrm{~kg}$ to $100 \mathrm{~kg}$. Each subject performs 4 sets of on-bed movement tests and 10 sets of bed-exit tests. In each on-bed movement test, the subject lies on his back and remains still on the mattress. After 25 seconds, the subject, then, follows turns over and remains still in the next 25 seconds. Finally, the subject changes back to lie on his back on mattress. Each subject repeats aforementioned steps three times in one test. In total, we acquire 12 groups of on-bed movement and 40 groups of bed exit. 
2) Sleep Event Detection Accuracy: The SleepSense system processes the acquired data from the aforementioned sets of tests with the framework in Section III-D. The result shows that the overall accuracy is $95.1 \%$, which we defined as the overall probability that the event is recognized correctly. The event detection confusion table is shown in Table II. In case of on-bed movement recognition, the SleepSense system successfully distinguishes 93 segments from the total 108 segments labeled with on-bed movement. In term of bed exit recognition, it recognizes 21 segments from the total 30 segments labeled with bed exit. In the case of breathing event, SleepSense identifies 405 segments from the total 408 segments. Noticeably, the classifier can distinguish the bed exit and breathing event precisely. No breathing events are recognized as the bed exit. It is the same for bed-exit recognition. This is because the features of the bed exit and breathing event vary dramatically in terms of time domain or frequency domain. However, the classification result for bed exit and on-bed movement event is inferior, because some of on-bed movements and bed-exit movements share the same features, such as the fast frequency and the large amplitude in the movement. There are also some errors for the on-bed movement and breathing event recognition, which is caused by the windowing issue. Specifically, because we employ the fixed length window in the segmentation, a movement may be incorrectly divided into two segments and loses the on-bed movement features, leading to the misclassification case.

TABLE II. THE CONFUSION MATRIX FOR THE SHORT-TERM STUDY.

\begin{tabular}{|c|c|c|c|c|}
\hline & $\begin{array}{c}\text { On-bed } \\
\text { movement }\end{array}$ & Bed exit & Breathing event & Precision \\
\hline $\begin{array}{c}\text { On-bed } \\
\text { movement }\end{array}$ & 93 & 10 & 5 & $88.6 \%$ \\
\hline Bed exit & 9 & 21 & 0 & $67.7 \%$ \\
\hline Breathing event & 3 & 0 & 405 & $98.8 \%$ \\
\hline Recall & $86.1 \%$ & $70.0 \%$ & $99.3 \%$ & \\
\hline
\end{tabular}

3) Breathing Rate Extraction: We verify the proposed breathing rate extraction algorithm by employing it on a short breathing signal, which is described in Fig. 7. It is an except from a relative long breathing signal. From the demodulated signal, we can observe that the breathing number is 21 during the 60 seconds. By applying the peak detection algorithm with adaptive threshold, we can obtain the relationship between breathing numbers and threshold values in the top of Fig. 7 . According to the algorithm, we look for the most flat region on the signal, which is zoomed in the upper part. The most flat region is circled by the red rectangle and the corresponding breathing number is 21 . Because the breathing rate algorithm employs different threshold values to calculate the number of peaks. Therefore, this algorithm can detect different values of peaks based on different threshold values. The red dots, as shown in the bottom of Fig. 7, are a group of detected peaks based on specific threshold values.

\section{Real-case study}

We also perform a long-term and real-world case study. This 75-minute nap experiment evaluates the wide usability and high accuracy of SleepSense in the real world.

1) The 75-minute Nap Study: The data are collected during a nap of one subject. Similarly, we deploy airflow sensor and acceleration sensor on the subject to establish the ground

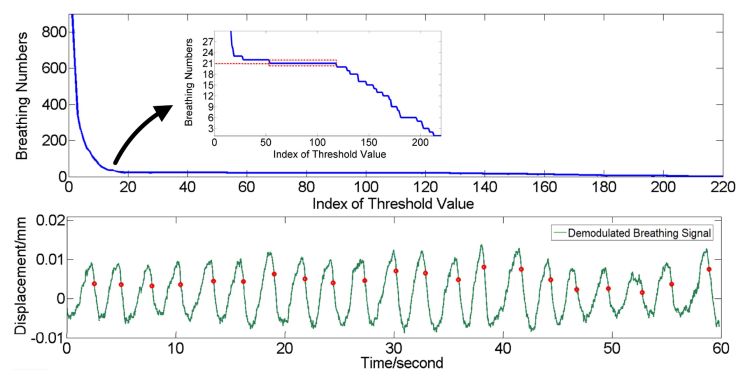

Fig. 7. The top sub-figure is the relationship between the detected breathing number and threshold value index. The bottom sub-figure is the detected peaks using a specific threshold value.

truth for breathing and movement signal. The displacement signal is shown in Fig. 8(a). The sleep event recognition framework frames the 75-minute signal into small segments. The ground truth in Fig. 8(b) shows that it has a total of 4 on-bed movements and 1 bed exit. Others are the breathing events. Because the duration of the on-bed movement exceeds the frame length, which is 5.12 seconds, two consecutive frames, thus, contain one movement signal. Therefore, there are total 8 frames that labeled as the on-bed movement event. The total number of breathing event frames is 869. Only 1 frame is labeled as the bed exit event. Finally, the trained sleep event detector in section IV-B processes all these frames. These segments will be classified into three sleep events: onbed movement, bed exit, or breathing event. Considering that

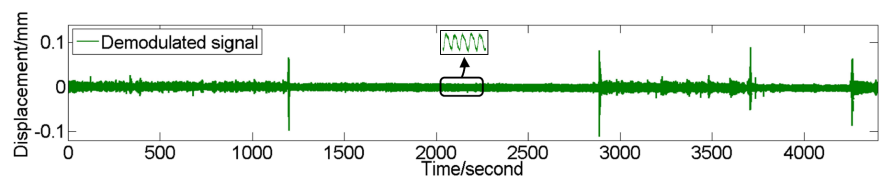

(a) The demodulated 75-minute displacement signal from SleepSense.

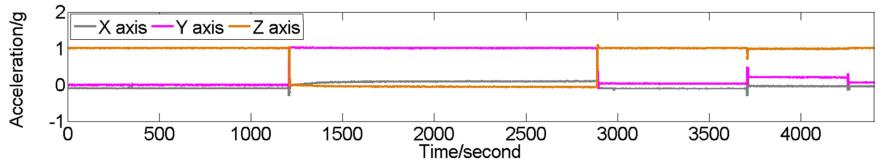

(b) The movement signal from the accelerometer sensor.

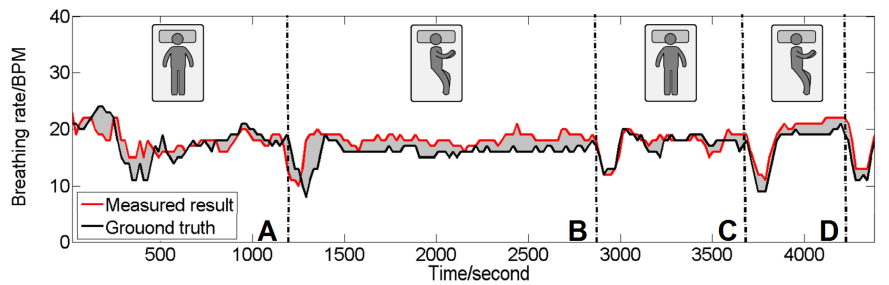

(c) The breathing rate curve of the 75-minute long-term monitoring data. The gray area is the error.

Fig. 8. (a) is the demodulated signal (75 minutes) from the SleepSense system; (b) is the ground truth provided by accelerometer; (c) is the breathing rate for this 75-minute nap with the airflow sensor as the ground truth signal.

there is only one bed-exit event, we focus on the evaluation of on-bed movement and breathing event detection. The result shows that the overall accuracy rate for sleep event detection is $99.54 \%$. Specifically, in this test, SleepSense distinguishes 868 breathing event frames. We note that the recognition rate for on-bed movement event detection is relatively lower, because of the two misclassified on-bed movement frames. The misclassification cases may be caused by the aforementioned 
windowing issue.

Moreover, we calculate the breathing rate by averaging the breathing counts in the previous 20 windows. Fig. $8(\mathrm{c})$ demonstrates the overall breathing rate for this short period of sleep. Specifically, the red curve is the estimated result by using the SleepSense system. The black curve is the ground truth signal. These two curves are matched similarly. Both are about 18 breath per minute (BPM). We can observe that the breathing rate varies during sleep in Fig. 8(c). When the subject is going to fall asleep, the breathing rate is more irregular, which changes between $20 \mathrm{BPM}$ and $10 \mathrm{BPM}$ in region $A$. However, the breathing rate becomes stable when the subject falls asleep. At the end of sleep, the breathing rate experiences another level changing. An interesting phenomenon is that when the subject changes sleep position, it is associated with a reducing breathing rate.

Furthermore, we quantify the error between the SleepSense-measured result and the ground truth signal by using the area under the curve (AUC) method as shown in Eq. (3):

Error Rate $=\frac{\mid A U C(\text { SleepSense })-A U C(\text { GroundTruth }) \mid}{A U C(\text { GroundTruth })}$.

We define the overall error rate as the ratio of total area difference between the SleepSense-measured result and ground truth to the total ground truth area. The overall error rate is only $6.65 \%$, which is the gray area in Fig. 8(c).

The error is caused by two factors: the on-bed movement and side sleep position. Fig. 8(c) shows a rising error rate (grey area) near the location where the subject changes to a different sleep position. This is because when an on-bed movement occurs, it is difficult for SleepSense to extract the breathing rate from that frame. However, the SleepSense will use the breathing number in the preceding frame that has no on-bed movement signal instead, therefore, causing the increased error rate. Another factor is the sleep position as seen from Fig. 8(c). The subject changes the sleep position from supine to side sleep and side sleep to supine four times during the nap. The error rate varies for different sleep positions. Specifically, the error rate is considerably lower when the subject is in the supine position. When subject changes the sleep position to the side, the chest displacement is less significant, and the breathing signal become relatively weak for the subject who is in side sleep position. The performance of the breathing rate extraction algorithm diminishes correspondingly.

\section{CONCLUSion AND Future Work}

In this paper, we present the SleepSense, a contactless and low-cost sleep monitoring system, which discriminates various sleep events and extracts the breathing rate accurately. In the implementation of SleepSense, we propose a novel signal processing framework for sleep event recognition, which contains feature extraction and the sleep event classification. A novel breathing rate extraction algorithm is devised to calcuate the breathing rate. We further empirically demonstrated the effectiveness of SleepSense in a short-term controlled study and a long-term real-case study. SleepSense can effectively identify the on-bed movement, bed exit, breathing event and extract the breathing rate with a high accuracy rate and wide usability. In the future, we will address the encountered issues such as the segmentation issue and side sleep position issue to improve the performance of SleepSense. Moreover, we will build a personalized sleep monitoring system using the acquired breathing pattern and movement distribution pattern provided by SleepSense.

\section{REFERENCES}

[1] N. Douglas, D. White, C. Pickett, J. Weil, and C. Zwillich. Respiration during sleep in normal man. Thorax, 37(11):840-844, 1982.

[2] A. Droitcour, V. Lubecke, J. Lin, and O. Boric-Lubecke. A microwave radio for doppler radar sensing of vital signs. In Microwave Symposium Digest, 2001 IEEE MTT-S International, volume 1, pages 175-178. IEEE, 2001.

[3] T. Hao, G. Xing, and G. Zhou. isleep: unobtrusive sleep quality monitoring using smartphones. In Proceedings of the 11th ACM Conference on Embedded Networked Sensor Systems, page 4. ACM, 2013.

[4] M.-C. Huang, J. Liu, W. Xu, C. Gu, C. Li, and M. Sarrafzadeh. A self-calibrating radar system design for measuring vital signs. 2015.

[5] E. Hughes, M. Lewis, and E. Reid. The application of speech recognition techniques to radar target doppler recognition: A case study. 2006.

[6] Y. Li, C. Gu, T. Nikoubin, and C. Li. Wireless radar devices for smart human-computer interaction. In Circuits and Systems (MWSCAS), 2014 IEEE 57th International Midwest Symposium on, pages 65-68. IEEE, 2014.

[7] J. Liu, M.-C. Huang, W. Xu, X. Zhang, L. Stevens, N. Alshurafa, and M. Sarrafzadeh. Breathsens: A continuous on-bed respiratory monitoring system with torso localization using an unobtrusive pressure sensing array. 2014.

[8] J. J. Liu, W. Xu, M.-C. Huang, N. Alshurafa, M. Sarrafzadeh, N. Raut, and B. Yadegar. A dense pressure sensitive bedsheet design for unobtrusive sleep posture monitoring. In Pervasive Computing and Communications (PerCom), 2013 IEEE International Conference on, pages 207-215. IEEE, 2013.

[9] L. Liu, M. Popescu, M. Skubic, M. Rantz, T. Yardibi, and P. Cuddihy. Automatic fall detection based on doppler radar motion signature. In Pervasive Computing Technologies for Healthcare (PervasiveHealth), 2011 5th International Conference on, pages 222-225. IEEE, 2011.

[10] O. B. Lubecke, P.-W. Ong, and V. Lubecke. 10 ghz doppler radar sensing of respiration and heart movement. In Bioengineering Conference, 2002. Proceedings of the IEEE 28th Annual Northeast, pages 55-56. IEEE, 2002.

[11] M. Rofouei, M. Sinclair, R. Bittner, T. Blank, N. Saw, G. DeJean, and J. Heffron. A non-invasive wearable neck-cuff system for real-time sleep monitoring. In Body Sensor Networks (BSN), 2011 International Conference on, pages 156-161. IEEE, 2011.

[12] M. Sateia, K. Doghramji, P. Hauri, and C. Morin. Evaluation of chronic insomnia. An American Academy of Sleep Medicine Review, 23(2):243308,2000 .

[13] C. Wang, A. Hunter, N. Gravill, and S. Matusiewicz. Unconstrained video monitoring of breathing behaviour and application to diagnosis of sleep apnoea. IEEE, 2014.

[14] J. Wang, X. Wang, L. Chen, J. Huangfu, C. Li, and L. Ran. Noncontact distance and amplitude-independent vibration measurement based on an extended dacm algorithm. Instrumentation and Measurement, IEEE Transactions on, 63(1):145-153, 2014.

[15] J. Wilde-Frenz and H. Schulz. Rate and distribution of body movements during sleep in humans. Perceptual and motor skills, 56(1):275-283, 1983.

[16] M. Zakrzewski, H. Raittinen, and J. Vanhala. Comparison of center estimation algorithms for heart and respiration monitoring with microwave doppler radar. Sensors Journal, IEEE, 12(3):627-634, 2012.

[17] C. Zheng, T. Hu, S. Qiao, Y. Sun, J. Huangfu, and L. Ran. Doppler bio-signal detection based time-domain hand gesture recognition. In Microwave Workshop Series on RF and Wireless Technologies for Biomedical and Healthcare Applications (IMWS-BIO), 2013 IEEE MTT-S International, pages 3-3. IEEE, 2013. 